Int. J. Odontostomat., 9(2):313-319, 2015.

\title{
Cáncer Oromaxilofacial en Niños: Parte I Tumores Malignos de Tejido Duro
}

\author{
Oromaxillofacial Cancer in Children: Part I Hard Tissue Malignant Tumors
}

\author{
Francisca Becerra*; Javiera Rehbein”; Alejandra Fernández ${ }^{* *}$ Maureen Marshall** \& Alfredo Esguep ${ }^{* * *}$
}

BECERRA, F.; REHBEIN, J.; FERNÁNDEZ, A.; MARSHALL, M. \& ESGUEP, A. Cáncer oromaxilofacial en niños: Parte I tumores malignos de tejido duro. Int. J. Odontostomat., 9(2):313-319, 2015.

RESUMEN: Las enfermedades malignas de cabeza y cuello en la población pediátrica son poco frecuentes. Considerando todos los tumores de cabeza y cuello, tanto benignos como malignos, éstos están representados por una frecuencia entre un $2 \%$ a $5 \%$ de todos los tumores pediátricos. A nivel de los maxilares, los tumores malignos en niños está representado por un rango que varía entre un $7 \%$ a $51 \%$. En Chile, se estiman menos de 500 casos nuevos por año y en el rango entre los 5 y 15 años constituyen la segunda causa de muerte, precedida por los accidentes, traumatismos y violencias. Es importante considerar, al estudiar este grupo de enfermedades, que existen diferencias con los tumores de adultos, tanto en su incidencia, biología, comportamiento, histología y manejo. Es por esta razón que nos parece necesario realizar una revisión de la literatura científica para obtener un perfil del cáncer bucomaxilofacial infantil, que aporte en el entendimiento y aplicación de programas adecuados en el contexto de la salud bucomaxilofacial. Adicionalmente, buscamos actualizar el conocimiento de estas patologías, caracterizándolas en relación a su epidemiología, etiopatogenia y tratamiento, de manera que contribuya a los cirujanos dentistas para realizar diagnósticos y la pronta derivación a un centro especializado de patología oral. Para un estudio preciso, esta revisión constará de tres partes: la primera es sobre "tumores malignos de tejido óseo", mencionando, entre ellos, al osteosarcoma, sarcoma de Ewing, linfomas tanto Hodgkin como No Hodgkin, fibrosarcoma e histiocitosis de células de Langerhans.

PALABRAS CLAVE: cáncer bucomaxilofacial, cáncer en niños, tumores malignos, tejido óseo.

\section{INTRODUCCIÓN}

Las enfermedades malignas de cabeza y cuello en la población pediátrica son poco frecuentes. Considerando todos los tumores de cabeza y cuello, tanto benignos como malignos, están representados por una frecuencia entre un $2 \%$ a $5 \%$ de todos los tumores pediátricos (Rahbar et al., 2006). A nivel de los maxilares, los tumores malignos en niños está representado por un rango que varía entre un $7 \%$ a $51 \%$ (Mohammed \& Aliyu, 2009). En Chile, se estiman menos de 500 casos nuevos por año (Campbell, 2005) y en el rango entre los 5 y 15 años constituyen la segunda causa de muerte, precedida por los accidentes, traumatismos y violencias (Vargas et al., 2011).

Es importante considerar al estudiar este grupo de enfermedades que existen diferencias con los tu- mores de adultos, tanto en su incidencia, biología, comportamiento, histología y manejo. Incluso cuando se diagnostican las mismas neoplasias en ambos grupos, se registran diferencias en su comportamiento clínico. Destaca que en el grupo pediátrico se han asociado a la génesis de tumores malignos la infección por virus Epstein - Barr y/o a los hábitos o costumbres maternas. Donde estos factores carcinogénicos podrían actuar en la vida intrauterina, lo que justifica que la mayoría de las neoplasias son de origen embrionario o sarcomatoso, a diferencia de lo que sucede en los adultos, donde existe una mayor tendencia a los tumores de origen epitelial (MacDonald, 2010).

Existe poca información disponible con respecto al manejo de los tumores en niños y generan preocupa-

\footnotetext{
* Cirujano Dentista, Práctica Privada, Viña del Mar, Chile.

" Cirujano Dentista, Docente Cátedra de Patología, Universidad Andrés Bello, Viña del Mar, Chile.

${ }^{* * *}$ Profesor titular Departamento de Cirugía y Patología, Facultad de Odontología, Universidad Andrés Bello, Viña del Mar, Chile.
} 
ciones adicionales en la elección del tratamiento, debido a que muchas veces es necesario el uso de quimioterapia y radioterapia, los que causan efectos adversos en el crecimiento y desarrollo de los niños, además de la carga afectiva que conlleva (Vargas et al.).

Nos parece necesario realizar una revisión de la literatura científica para obtener un perfil del cáncer bucomaxilofacial infantil, que aporte en el entendimiento y aplicación de programas adecuados en el contexto de la salud bucomaxilofacial. Adicionalmente, buscamos actualizar el conocimiento de estas patologías, caracterizándolas en relación a su epidemiología, etiopatogenia y tratamiento, de manera que contribuya a los cirujanos dentistas para realizar diagnósticos y la pronta derivación a un centro especializado de patología oral. Para un estudio preciso, esta revisión constará de tres partes: la primera se sobre "tumores malignos de tejido óseo". La segunda sobre "tumores malignos odontogénicos y de glándulas salivales". El tercero sobre "tumores malignos a nivel de tejido blando". En todos los apartados nos referiremos a los distintos tipos de neoplasias malignas más frecuentes a nivel bucomaxilofacial en la población pediátrica.

A continuación se describe la primera parte de ellas "tumores malignos óseos". Para una mejor comprensión y facilidad en el estudio de las neoplasias pediátricas malignas a nivel oral, se puede observar en la Tabla I una clasificación con las neoplasias más frecuentes, modificada de la Organización Mundial de la Salud del año 2002 y 2005.

Nos referiremos principalmente al Osteosarcoma, Sarcoma de Ewing y los Linfomas, debido a que son las neoplasias malignas del tejido óseo más frecuentes a nivel del grupo pediátrico.

a) Tumores osteogénicos: Osteosarcoma. El osteosarcoma es un tumor maligno primario intramedular de alto grado de malignidad, en el cual las células neoplásicas producen osteoide (Fletcher et al., 2002). Corresponde al tumor óseo de mayor frecuencia en niños y adultos jóvenes, de todo el organismo (Huh et al., 2012). En general se desarrolla aproximadamente en una de cada 100.000 personas. Se presenta comúnmente en huesos largos, no obstante es posible encontrarlo en la zona de cabeza y cuello, siendo los huesos maxilares los más involucrados en esta región (Huh et al.). En los maxilares sólo un $6 \%$ a un $20 \%$ han sido diagnosticados en la primera y segunda década de vida (Donaldson et al., 2004). Donaldson et al. reportan que existe cier- ta preferencia por el compromiso mandibular que maxilar. En la mándibula el sitio más afectado es el cuerpo y en el maxilar el reborde alveolar, paladar y seno (Clark et al., 1983). En relación al género, por un lado se reporta que es más frecuente en el sexo masculino (Donaldson et al.) y por otro que no existe predilección por sexo (Donaldson et al.).

Es importante nombrar que el osteosarcoma pediátrico muestra características clínicas distintas a la de los osteosarcomas de extremidades, e incluso a la de los osteosarcomas maxilares en adultos.

En cuanto a su etiología, no existe un factor desencadenante específico, pero se ha asociado a historia previa de radioterapia y predisposición genética por mutación del gen RB1, el que se asocia a la génesis del Retinoblastoma (Daw et al., 2000).

El tratamiento por lo general es multimodal e incluye resección quirúrgica con márgenes de seguridad, quimioterapia y radioterapia (Clark et al.). Muchas veces no es posible realizar la resección quirúrgica de la lesión en la región de cabeza y cuello, debido a complicaciones anatómicas, efecto mutilantes y complicaciones secundarias, por lo que en estos casos se recurre a tratamientos de quimioterapia y/o radioterapia.

Los osteosarcomas de los maxilares presentan mejor pronóstico que el de los huesos largos (Clark et al.). La tasa de sobrevida reportada varía entre un $10 \%$ a $37 \%$ a los 5 años (Daw et al.).

b) Sarcoma de Ewing o Tumor Neuroectodérmico primitivo. Se definen como sarcomas de células redondas que muestran diferentes grados de diferenciación neuroectodérmica. El término Sarcoma de Ewing, ha sido utilizado para aquellos tumores que carecen de diferenciación neuroectodérmica. Mientras que el término Tumor Neuroectodérmico primitivo, ha sido utilizado para aquellos tumores que demuestran características de diferenciación neuroectodérmica.

Corresponden al segundo tumor óseo maligno más frecuente en niños (Fletcher et al.), precedido por el osteosarcoma. Corresponde aproximadamente al $4-10 \%$ de las neoplasias óseas malignas y aproximadamente al $3 \%$ de los tumores malignos pediátricos (Karosas, 2010). En la región de cabeza y cuello representa al $1-5 \%$ de todos los casos de este tumor y los sitios más frecuentemente afectados corresponden a los huesos del cráneo y a los maxilares (Bakhshi 
BECERRA, F.; REHBEIN, J.; FERNÁNDEZ, A.; MARSHALL, M. \& ESGUEP, A. Cáncer oromaxilofacial en niños: Parte I tumores malignos de tejido duro. Int. J. Odontostomat., 9(2):313-319, 2015.

Tabla I. Clasificación modificada de la OMS sobre neoplasias malignas en niños (Barnes et al., 2005).

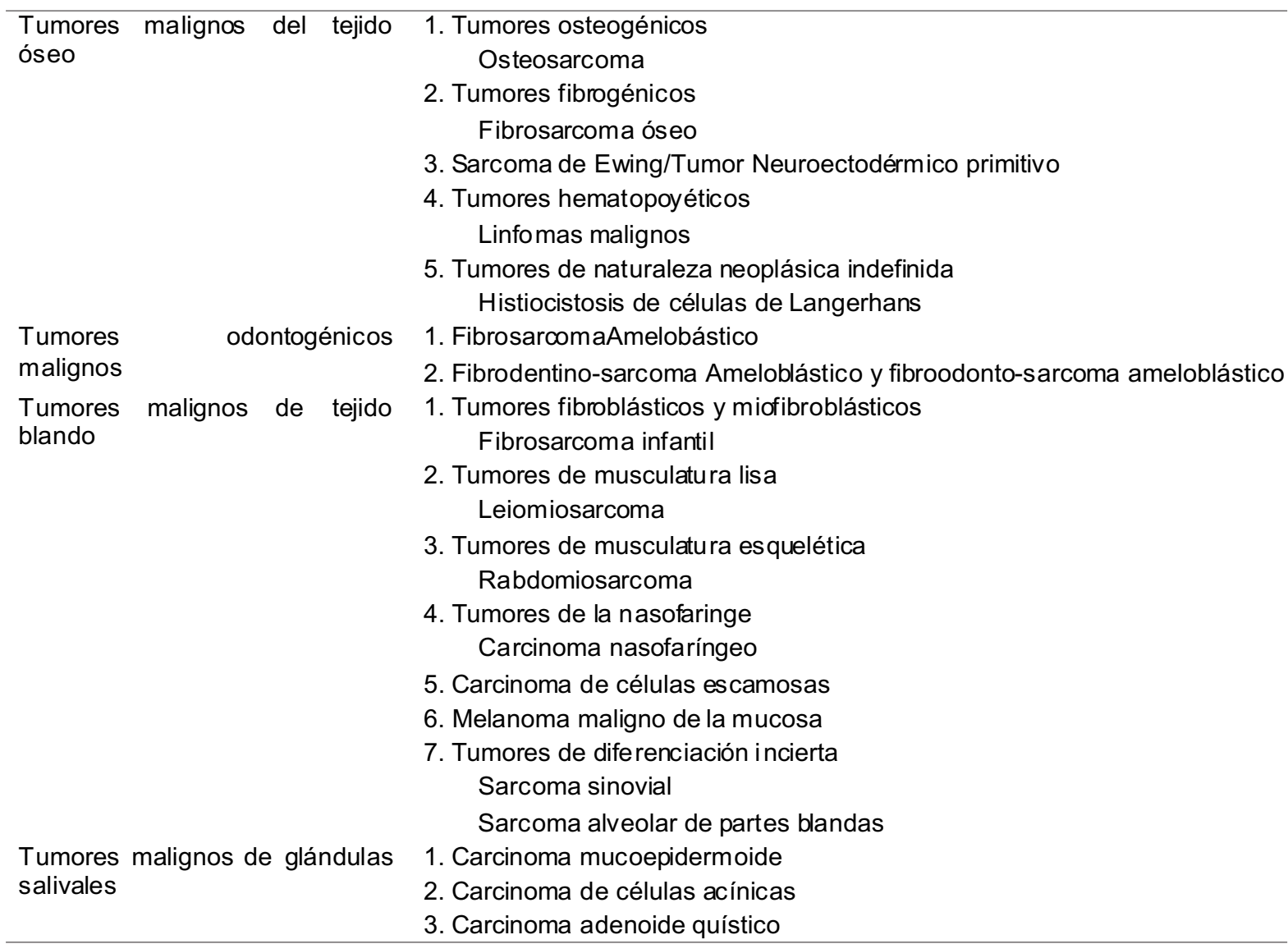

et al., 2011). Es preciso mencionar que existe mayor incidencia en la mandíbula, con predilección por la rama, que en el maxilar. Estos últimos presentan una incidencia aproximada de $1 \%$ en la población general (Neville et al., 2009). Es más frecuente en el sexo masculino y en caucásicos. Se estima que se desarrolla más frecuente en las primeras dos décadas de la vida. Aproximadamente el $80 \%$ de los pacientes afectados son menores de 20 años al momento del diagnóstico (Karosas).

El origen de este tumor de células redondas es controversial. Originalmente fue descrito como proveniente de células óseas indiferenciadas. Sin embargo, otros estudios sugieren que presenta un origen neuroectodérmico, derivado de tejido neural primitivo con variados grados de diferenciación. Cabe mencionar que las células tumorales presentan, en un $80 \%$ a $90 \%$ de los casos, una traslocación recíproca entre los cromosomas $(11 ; 22)$ ( q 24; q 12). Esta alteración lleva a la creación de una oncoproteína, con propiedades oncogénicas (Karosas).
El tratamiento es multimodal, consta de tratamiento quirúrgico, radioterapia y quimioterapia (Karosas). Esta modalidad ha permitido aumentar la tasa de supervivencia, de un $16 \%$ a un $75 \%$. Pero en general presentan un mal pronóstico, por sus características de presentar un rápido crecimiento y alto potencial de desarrollar metástasis (Gupta et al., 2011).

c) Tumores hematopoyéticos: Linfomas. Los linfomas son neoplasias compuestas de células linfoides malignas. En esta ubicación son poco comunes y representan aproximadamente el $7 \%$ de todas las neoplasias malignas óseas. Pueden afectar a cualquier edad, pero con una mayor tendencia a afectar a los adultos (Fletcher et al.). A pesar de ello, los linfomas son una de las neoplasias más comunes en niños, sobre todo en las regiones de África (Mohammed \& Aliyu). Corresponden aproximadamente al $10 \%$ de todas las neoplasias malignas en menores de 15 años y representan más del 50\% de los tumores malignos pediátricos de cabeza y cuello. Generalmente se presentan como un aumento de volumen a nivel de la región del cuello (Edwards, 2012). 
La mayoría se originan en los tejidos linfoides y sólo el $24 \%$ de ellos se desarrollan en sitios extraganglionares. Sin embargo, se puede presentar a nivel de la cavidad oral, donde los sitios comúnmente afectados son el paladar duro, gingiva, glándulas salivales y lengua (Otmani \& Khattab, 2008).

Clásicamente los linfomas son divididos en dos grupos, Linfoma de Hodgkin y Linfoma no Hodgkin y la OMS describe más de 50 subtipos histopatológicos distintos (Zapater et al., 2010).

d) Linfomas de Hodgkin. Corresponden a un desorden linfoproliferativo maligno, caracterizado por las células de Reed-Sternberg (Neville et al.). Cada año 5,5 millones menores de 15 años son afectados por esta enfermedad. En Estados Unidos representa el $4 \%$ de las neoplasias en menores de 16 años.

Su causa exacta es desconocida, pero se asocia a la infección por el Virus Epstein Bar (VEB) y a ciertas inmunodeficiencas. Aproximadamente el 40$50 \%$ de los linfomas están asociados al VEB en países desarrollados (Gaini et al., 2009). Es más frecuente en niños de sexo masculino, hasta 3 a 4 veces más que por el sexo femenino.

Existen cuatro subtipos de Linfomas de Hodgkin según las características de la población linfocitaria que presenten, lo que permite determinar la planificación del tratamiento y pronóstico de la enfermedad (Tabla II).

Para el tratamiento existen combinaciones de fármacos quimioterapéuticos específicos para cada subtipo, por su parte la radioterapia tiene un papel importante en el control de la enfermedad (Neville et al.). Este tipo de linfoma es un cáncer altamente curable y los casos pediátricos tienen una mejor tasa de respuesta frente al tratamiento que los adultos (Gaini et al.).

e) Linfomas no Hodgkin. Son tumores de células linfoides que a diferencia del tipo no Hodgkin carecen de células de Reed-Stermberg. Sin embargo, ambos grupos presentan un estadiaje clínico similar y diferentes subtipos histopatológicos, con variadas opciones de tratamiento y pronóstico. Es más fecuente en el sexo masculino (Neville et al.) y destaca por ser altamente agresivo en los niños por su rápido crecimiento. Consituye un $6 \%$ de todos los tumores pedíatricos, representan el $60 \%$ de todos los linfomas y el $8-10 \%$ de todas las neoplasias malignas en niños.
Es preciso mencionar que se subdividen en aquellos que derivan de células B y T. Por su parte los de presentación oral son predominantemente derivados de células B $(92 \%)$ y en menor frecuencia de células $\mathrm{T}(8 \%)$ (Otmani \& Khattab). El subtipo endémico más común, sobre todo en la población pediátrica de África es el linfoma de Burkitt.

Su etiología también es desconocida, pero se asocia a infección por VEB, por el virus herpes humano, por el virus linfotrópico humano de células $\mathrm{T}$, inmunodeficiencias congénitas, inmunodeficiencias inducidas por drogras y alteraciones géneticas (Otmani \& Khattab).

A diferencia de la mayoría de los tratamientos empleados para neoplasias, en este grupo el manejo quirúrgico no es frecuentemente indicado, por lo que se emplea radioterapia y quimioterapia. Desafortunadamente los rangos de curación no son elevados y la tasa de mortalidad es aproximadamente de un $50 \%$ a los 5 años de haberse diagnosticado e iniciado el tratamiento (Sapp et al., 2005).

f) Linfoma de Burkitt. Corresponde a una forma agresiva de linfoma no Hodgkin de células B, con la particularidad de ser una de las neoplasias malignas de crecimiento más rápido (Vargas et al.). Afecta típicamente a zonas extralinfoides, con predilección por los maxilares y las vísceras (Neville et al.). Es uno de los tumores más comunes en la población pediátrica y en los adultos jóvenes, alcanzando aproximadamente un 30-50\% de los linfomas pedíatricos en los países occidentales (Bi et al., 2012). En África ecuatorial la prevalencia tiende a ser mayor, posiblemente debido a los altos índices de malaria, virus de la inmunodeficiencia humana (VIH) y VEB (Park \& Chung, 2010). El compromiso maxilar no endémico alcanza del 7 al $29 \%$ de los casos (Neville et al.), preferentemente en pacientes de sexo masculino y caucásicos (Park \& Chung).

En relación a su etiología, se describen numerosos factores asociados, tales como causas genéticas, nivel socioeconómico, zonas geográficas, infección por el VEB y VIH, anomlías cromosómicas, defectos inmunes, alteraciones nutricionales como déficit proteico. Generalmente presenta traslocación de una porción del cromosoma 8 a los cromosomas 2, 14 y 22. Esto permite la activación del oncogen c-myc que es una proteína de transcripción de ADN que partipa en el inicio del ciclo celular, lo que es claramente un factor clave la oncogénesis (Neville et al.). 
Tabla II. clasificación y pronóstico del linfoma de Hodgkin (Neville et al., 2009).

\begin{tabular}{lcc}
\hline Tipo histológico & Pronóstico & $\begin{array}{c}\text { Supervivencia a } \\
\text { los } \mathbf{5} \text { años }\end{array}$ \\
\hline Predominio linfocítico & Muy bueno & $90 \%$ \\
Esclerosis nodular & Bueno & $70 \%$ \\
Celularidad mixta & Regular & $60 \%$ \\
Depleción linfocítica & Malo & $20 \%$ \\
\hline
\end{tabular}

En cuanto al tratamiento, el manejo quirúrgico se utiliza sólo para la realización de las biopsias, por lo que el tratamiento considera el uso de quimioterapia y radioterapia (Neville et al.).

g) Tumores fibrogénicos: Fibrosarcoma óseo. Es un tumor maligno primario de células fusiformes producido en el hueso, poco frecuente y constituye hasta un $5 \%$ de todos los tumores malignos primarios.

La OMS establece que la etiología es desconocida, pero se ha asociado a diversas condiciones como tratamientos previos de radioterapia, tumores de células gigantes, osteocondroma, osteomielitis crónicas, infartos óseos, displasia fibrosa, fibroma amelobástico y displasia ósea hereditaria.

El tratamiento puede consistir en la resección total de la lesión, sumado o no a radioterapia y quimioterapia. Su tasa de supervivencia varía entre un $34 \%$ y $83 \%$ a los 10 años (Fletcher et al.).

h) Tumores de naturaleza neoplásica indefinida: Histiocitosis de células de Langerhans (HCL). HCL es una proliferación neoplásica de células de Langerhans (Fletcher et al.), poco común, de comportamiento agresivo. Es una afección que predomina en la infancia, donde más del $50 \%$ de los niños afectados son menores de 15 años. Afecta aproximadamente a 5 niños y 1,2 adultos por cada un millón de persona. Generalmente afecta la región de cabeza y cuello $(80 \%)$ y en un $10 \%$ a nivel oral.

Se sugiere que presenta una relación directa con un sistema inmune inmaduro o con un estado infeccioso.

Tanto el tratamiento como el pronóstico varían dependiendo de la severidad de la presentación clínica. Las modalidades terapéuticas varían desde un curetaje a exicisión quirúrgica de la lesión, con o sin quimioterapia o radioterapia coadyuvantes (Park \& Chung).

\section{DISCUSIÓN}

A nivel mundial, la información a cerca de tumores malignos del tipo maxilofacial es limitada y consiste principalmente en datos en la población adulta. Consultamos las bases de datos de PubMed, CENTRAL y de la Universidad Andrés Bello y la mayoría de los estudios encontrados se refieren a casos de tumores orales y maxilofaciales específicos, y otros suelen agrupar casos pediátricos junto con los casos de adultos. A pesar de la baja frecuencia que presenta este grupo de patologías, no es menos cierto que es necesario saber con precisión cuales son los tejidos orales desde los cuales se pueden originar neoplasias malignas en los niños y tener una breve referencia diferencial entre ellos.

Es difícil abordar el tema desde el punto de vista de una revisión bibliográfica, ya que no se cuenta con estudios sistematizados de todas las manifestaciones posibles de cáncer en la cavidad oral al margen de su poca frecuencia. Esto dificulta el análisis epidemiológico, que actualmente permite entender los patrones de desarrollo de cada una de las neoplasias y establecer políticas orientadas al fomento de la prevención y diagnóstico precoz.

Datos tales como incidencia, prevalencia, tipos y distribución de estas lesiones según edad, género, localización anatómica, entre otras variables, provienen de estudios aislados, por lo que es incierto si las series disponibles de estas patologías reflejan de forma fidedigna tanto su verdadera naturaleza como sus características clinicopatológicas, por lo que no es posible hacer consideraciones definitivas en torno a ello.

Además, al estudiar este grupo es importante considerar la distribución geográfica. La mayoría de países occidentales reportan una baja incidencia de tumores malignos orales en el grupo pediátrico. Por otro lado, en África, se observa una mayor prevalen- 
cia de tumores malignos, lo que se atribuye a un elevado porcentaje de linfomas, asociado probablemente a la alta prevalencia de malaria, VIH y virus Epstein Barr. Esta variación también puede ser aplicada a las distintas zonas de nuestro país, tema relevante de ser estudiado. Cabe destacar que en Chile no existen estadísticas sobre la casuística de estas patologías a nivel bucomaxilofacial en los niños. Pero si en la última publicación del MINSAL existen alusiones sobre cáncer oral en el grupo pediátrico, donde mencionan principalmente el rabdomiosarcoma, linfoma de Hodgkin y la Histiocitosis. Desde un punto de vista especializado es necesario tener una clara idea de todas las pontencialidades cancerológicas de la boca. Por otro lado, para favorecer su diagnóstico creemos necesario que los laboratorios de histopatología cuen- ten con herramientas inmunohistoquímicas de marcadores tumorales y también abrir la posibilidad de establecer relaciones con laboratorios de citogenética e imagenología.

El amplio espectro de patologías encontradas requiere de atención y estrecha colaboración entre pediatras oncólogos, radioterapeutas, patólogos orales y odontólogo, todos ellos especializados en el área de cabeza y cuello.

Esperamos que esta revisión constituya un instrumento que permita mejorar el conocimiento de estas lesiones y que sea un complemento a la guía "Cuando sospechar de un cáncer en el niño y cómo derivar", publicada por el MINSAL el año 2011.

BECERRA, F.; REHBEIN, J.; FERNÁNDEZ, A.; MARSHALL, M. \& ESGUEP, A. oromaxillofacial cancer in children: part I hard tissue malignant tumors. Int. J. Odontostomat., 9(2):307-312, 2015.

SUMMARY: Malignant head and neck disease in the pediatric population are rare. Considering all head and neck tumors, both benign and malignant, they represent between $2 \%$ to $5 \%$ of all pediatric tumors. At the level of the jaws, malignant tumors in children present in a range that varies from $7 \%$ to $51 \%$. In Chile, fewer than 500 new cases are reported each year and in the 5 to 15 year-range are estimated to constitute the second cause of death, preceded by accidents, injuries and violence. When studying this group of diseases it is important to consider the differences with adult tumors, both in incidence, biology, behavior, histology and management. Therefore we believe it necessary to conduct a review of scientific literature to obtain a profile of child oral maxillofacial cancer, contributing to the understanding and implementation of appropriate programs in the context of maxillofacial health. In addition, we seek to update knowledge of these pathologies, characterizing their epidemiology, pathogenesis and treatment, so as to contribute to dental surgeons for diagnosis and early referrals to specialized centers for oral pathology. For a detailed study, this review will consist of three parts: the first is on "malignant bone tumors", included among these are: osteosarcoma, Ewing's sarcoma, Hodgkin's lymphoma, non-Hodgkin's lymphoma, Langerhans cell histiocytosis and fibrosarcoma.

KEY WORDS: maxillofacial cancer, childhood cancer, malignant tumors, bone tissue.

\section{REFERENCIAS BIBLIOGRÁFICAS}

Bakhshi, S.; Pathania, S.; Mohanti, B. K.; Thulkar, S. \& Thakar, A. Therapy and outcome of primitive neuroectodermal tumor of the jaw. Pediatr. Blood Cancer, 56(3):477-81,2011.

Barnes, L.; Eveson, J. W.; Reichart, P. \& Sidransky, D. Pathology \& Genetics. Head and Neck Tumours. Albany, World Health Organization Classification of Tumours. IARC Press, 2005.

Bi, C. F.; Tang, Y.; Zhang, W. Y.; Zhao, S.; Wang, X. Q.; Yang, Q. P.; Li, G. D. \& Liu, W. P. Sporadic Burkitt lymphomas of children and adolescents in Chinese: a clinicopathological study of 43 cases. Diagn. Pathol., 7:72, 2012.

Campbell, M. Desarrollo de la Oncología Pediátrica en Chile. Rev. Pediatr. Electron., 2(2):1-4, 2005.
Clark, J. L.; Unni, K. K.; Dahlin, D. C. \& Devine, K. D. Osteosarcoma of the jaw. Cancer, 51(12):2311-6, 1983.

Daw, N. C.; Mahmoud, H. H.; Meyer, W. H.; Jenkins, J. J.; Kaste, S. C.; Poquette, C. A.; Kun, L. E.; Pratt, C. B. \& Rao, B. N. Bone sarcomas of the head and neck in children: the St Jude Children's Research Hospital experience. Cancer, 88(9):2172-80, 2000.

Donaldson, M. E.; Geist, J. R. \& Daley, T. D. Osteosarcoma of the jaws in children. Int. J. Paediatr. Dent., 14(1):5460, 2004.

Edwards, S. P. Pediatric Malignant Tumours of the Head and Neck. En: Current Therapy In Oral And Maxillofacial Surgency. Chapter 93. St. Louis, Elsevier, 2012. pp.82082. 
Fletcher, C. D. M.; Unni, K. K. \& Mertens, F. Pathology and Genetics of Tumours of Soft Tissue and Bone. Lyon, World Head Organization Classification of Tumours, IARC Press, 2002.

Gaini, R. M.; Romagnoli, M.; Sala, A. \& Garavello, W. Lymphomas of head and neck in pediatric patients. Int. J. Pediatr. Otorhinolaryngol., 73 (Suppl. 1):S65-70, 2009.

Gupta, S.; Gupta, O. P.; Mehrotra, S. \& Mehrotra, D. Ewing sarcoma of the maxilla: Arare presentation. Quintessence Int., 40(2):135-40, 2011.

Huh, W. W.; Holsinger, F. C.; Levy, A.; Palla, F. S. \& Anderson, P. M. Osteosarcoma of the jaw in children and young adults. Head Neck, 34(7):981-4, 2012.

Karosas, A. O. Ewing's sarcoma. Am. J. Health Syst. Pharm., 67(19):1599-605, 2010.

MacDonald, T. Pediatric cancer: Pediatric Cancer: A Comprehensive Review. Part I: Biology, Epidemiology, Common Tumours, Principles of Treatment and Late Effects. Can. Pharm. J., 143(4):176-83, 2010.

Mohammed, A. \& Aliyu, H. O. Childhood cancers in a referral hospital in northern Nigeria. Indian J. Med. Paediatr. Oncol., 30(3):95-8, 2009.

Neville, B. W.; Damm, D. D.; Allen, C. M. \& Bouquot, J. Oral and Maxilofacial Pathology. 2a ed. Philadelphia, Saunders, 2001.

Otmani, N. \& Khattab, M. Oral Burkitt's lymphoma in children: the Moroccan experience. Int. J. Oral Maxillofac. Surg., 37(1):36-40, 2008.

Park, J. W. \& Chung, J. W. Long-term treatment of Langerhans cell histiocytosis of the mandibular condyle with indomethacin. Oral Surg. Oral Med. Oral Pathol. Oral Radiol. Endod., 109(4):e13-21, 2010.

Rahbar, R.; Grimmer, J. F.; Vargas, S. O.; Robson, C. D.; Mack, J. W.; Perez-Atayde, A. R.; Marcus, K. J.; Grier, H. E.; Healy, G. B. \& McGill, T. J. Mucoepidermoid carcinoma of the parotid gland in children: A 10-year experience. Arch. Otolaryngol. Head Neck Surg., 132(4):375-80, 2006.

Sapp, J. P.; Eversole, L. R. \& Wysocki, G. P. Patología Oral y Maxilofacial Contemporánea. $2^{\mathrm{a}}$ ed. Barcelona, Elservier Mosby, 2005.

Vargas, L.; Varas, M.; Campbell, M.; Salgado, C.; Fuentes, P.; Becker, A.; Neira, L.; Derio, L. \& Sepúlveda, F. Cuando sospechar un cáncer en el niño y cómo derivar. $4^{a}$ ed. Santiago de Chile, Subsecretaría de Salud Pública, Ministerio de Salud, Gobierno de Chile, 2011.
Zapater, E.; Bagán, J. V.; Carbonell, F. \& Basterra, J. Malignant lymphoma of the head and neck. Oral Dis., 16(2):119-28, 2010.

Direccion para Correspondencia:

Alfredo Esguep Sarah

Avenida Valparaíso 1560

Viña del Mar

CHILE

Email: aesguep@unab.cl

Recibido: 29-09-2014

Aceptado: 26-03-2015 